\title{
Longitudinal and transverse $\Lambda$ polarization at COMPASS
}

\author{
Donghee Kang ${ }^{* \dagger}$ \\ on the behalf of the COMPASS collaboration \\ Helmholtz Institut Mainz, Johannes Gutenberg-Universität Mainz \\ Johann-Joachim-Becher-Weg 45, 55099 Mainz, Germany \\ E-mail: donghee.kang@cern.ch
}

\begin{abstract}
At the COMPASS experiment $\Lambda$ and $\bar{\Lambda}$ particles are produced with high statistics in deep inelastic scattering (DIS) processes of $160 \mathrm{GeV} / \mathrm{c}$ polarized muons. Since both, beam and target, are polarized, various studies on the $\Lambda$ polarization are possible. We present results on the longitudinal polarization transfer from muons to $\Lambda$ hyperons produced by scattering off an unpolarized isoscalar target and preliminary results on the transverse $\Lambda$ polarization with a transversely polarized proton target. The $\Lambda$ and $\bar{\Lambda}$ polarization can be studied by measuring the acceptance corrected angular distribution of its decay products. The longitudinal spin transfers to $\Lambda$ and $\bar{\Lambda}$ produced in the current fragmentation region exhibit different behaviours as a function of $x_{B j}$ and $x_{F}$. The $x_{B j}$ and $x_{F}$ dependences of $\Lambda$ polarization are compatible with zero, while $\bar{\Lambda}$ polarization tends to increase with $x_{F}$. Information on the transversely polarized quark distributions $\Delta_{T} q(x)$ in the nucleon can be accessed by measuring the transverse $\Lambda$ and $\bar{\Lambda}$ polarizations, which refer to a spin correlation between the transversely polarized nucleons and $\Lambda \mathrm{s}$. The transverse polarization is measured as function of $x_{B j}$ and $z$ and found to be compatible with zero.
\end{abstract}

XVIII International Workshop on Deep-Inelastic Scattering and Related Subjects, DIS 2010 April 19-23, 2010

Firenze, Italy

\footnotetext{
${ }^{*}$ Speaker.
} 


\section{Introduction}

The production of hyperons in polarized Deep Inelastic Scattering (DIS) is a powerful tool to provide new information on the unpolarized quark distributions $q\left(x_{B j}\right)$ and on the helicity distributions $\Delta q\left(x_{B j}\right)$, and on the fragmentation process of quarks. In a simple parton model a polarized lepton interacts preferentially with a quark polarized oppositely to the nucleon. The struck quark from a DIS reaction hadronizes with a certain probability into a $\Lambda$ hyperon and transfers a fraction of its polarization to the $\Lambda$. Thus the measurement of the spin transfer to the $\Lambda$ hyperon or the spin correlation between initial quark and the $\Lambda$ hyperon provides an insight to $q\left(x_{B j}\right)$ and $\Delta q\left(x_{B j}\right)$ in the nucleon. For the kinematic conditions of the COMPASS experiment the role of these effects has been investigated with the longitudinal and transverse polarization of $\Lambda$ and $\bar{\Lambda}$ hyperons [1]. In semi-inclusive DIS, the longitudinal $\Lambda$ polarization is expressed by [2]

$$
P_{\Lambda}\left(x_{B j}, z\right)=\frac{\sum_{q} e_{q}^{2}\left[P_{B} D_{L}(y) q\left(x_{B j}\right)+f P_{T} \Delta q\left(x_{B j}\right)\right] \cdot \Delta D_{q}^{\Lambda}(z)}{\sum_{q} e_{q}^{2}\left[q\left(x_{B j}\right)+P_{B} D_{L}(y) f P_{T} \Delta q\left(x_{B j}\right)\right] \cdot D_{q}^{\Lambda}(z)},
$$

where $e_{q}$ is the charge of quarks and anti-quarks, $f$ is the target dilution factor, and $P_{B}$ and $P_{T}$ are the beam and target polarization, respectively. The factor $D_{L}(y)=1-(1-y)^{2} / 1+(1-y)^{2}$ is the virtual photon depolarization factor. When only the beam is polarized and the target is not polarized, a possible correlation between the struck quark and the $\Lambda$ hyperon can be expressed by the spin transfer $D_{L L}=P_{\Lambda} / P_{B} D(y)$. The essential ingredient to the spin transfer is the fraction of polarized and unpolarized fragmentation functions $\Delta D_{q}^{\Lambda}(z) / D_{q}^{\Lambda}(z)$ for different quark flavour. If the $\Lambda$ is produced with a longitudinally polarized target, measurement of the spin transfer to hyperons can be used to determine the polarized quark distributions $\Delta q\left(x_{B j}\right)$ and $\Delta \bar{q}\left(x_{B j}\right)$ in the nucleon.

The measurement of the so-called transversity distribution functions $\Delta_{T} q\left(x_{B j}\right)$ in the nucleon is an important physics program of the COMPASS experiment. A promising channel for the measurement of transversity in SIDIS is the transverse $\Lambda$ polarization by means of spin correlation between transversely polarized initial quark and $\Lambda$ hyperons [3]. The transverse polarization of the $\Lambda$ is given by [4]

$$
P_{\Lambda}\left(x_{B j}, z\right)=f P_{T} D(y) \frac{\sum_{q} e_{q}^{2} \Delta_{T} q\left(x_{B j}\right) \Delta_{T} D_{q}^{\Lambda}(z)}{\sum_{q} e_{q}^{2} q\left(x_{B j}\right) D_{q}^{\Lambda}(z)}
$$

where the depolarization factor for transversely polarized target is $D(y)=2(1-y) / 1+(1-y)^{2}$. It appears that $\Delta_{T} q\left(x_{B j}\right)$ is coupled to $\Delta_{T} D_{q}^{\Lambda}(z)$. By measuring $P_{\Lambda}\left(x_{B j}, z\right)$ in different bins of $x_{B j}$ and $z$, one can access $\Delta_{T} q\left(x_{B j}\right)$ when modeling $\Delta_{T} D_{q}^{\Lambda}(z)$, or try to disentangle both $\Delta_{T} q\left(x_{B j}\right)$ and $\Delta_{T} D_{q}^{\Lambda}(z)[$ [ß], but a large statistics is needed.

\section{Measurement of $\Lambda$ and $\bar{\Lambda}$ polarization}

The results obtained for the longitudinal lambda polarization are based on the data taken during the 2003-2004 run recording with two oppositely polarized deuteron target cells oriented longitudinallly with respect to the muon beam. For transverse $\Lambda$ polarization, the analysis has been done from the data recorded during the 2007 run with a transversely polarized proton target, which consists of three oppositely polarized target cells. 
DIS events are selected by requiring the momentum transferred of the virtual photon to be $Q^{2}>$ $1(\mathrm{GeV} / c)^{2}$. $\Lambda^{\prime}$ 's and $\bar{\Lambda}$ 's are identified from their weak decay $\Lambda \rightarrow p \pi^{-}$and $\bar{\Lambda} \rightarrow \bar{p} \pi^{+}$. A cut to the target volume has been applied in order to ensure an equal beam flux in the different target cells. Only tracks with momenta larger than $1 \mathrm{GeV} / c$ were selected to provide good tracking efficiency. A collinearity angle between the lambda direction calculated by the position of the primary and secondary vertex and reconstructed $\Lambda$ from the decay prod-
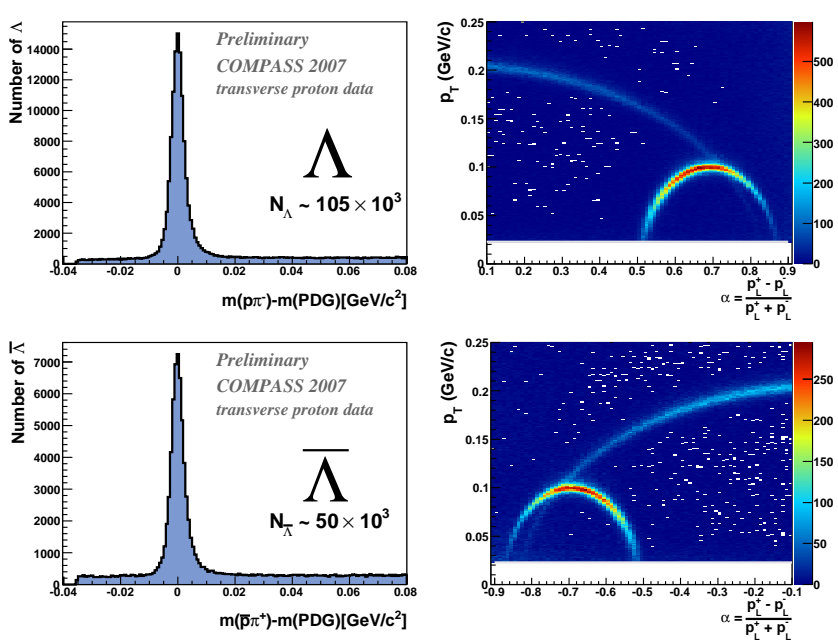

Figure 1: Invariant mass distribution and corresponding Armenteros-Podolanski plots for $\Lambda$ and $\bar{\Lambda}$. ucts have to be within $10 \mathrm{mrad}$. The contamination from $e^{+} e^{-}$pairs from photon conversion is reduced by requiring a minimal transverse momentum $p_{T}>23 \mathrm{MeV} / c$ of hadrons with respect to the reconstructed $\Lambda$ momentum. The last step to reduce the remaining background is to employ information from the RICH detector. Particle identification is only applied to hadrons in the measurement of the transverse $\Lambda$ polarization. Since the momentum threshold to identify a proton is high and thus limiting the available statistics significantly, a veto condition is used instead. The veto condition is based on a likelihood method and rejects $e^{+}\left(e^{-}\right), \pi^{+}\left(\pi^{-}\right)$and $K^{+}\left(K^{-}\right)$particles from the proton (anti-proton) candidates in the $\Lambda(\bar{\Lambda})$ decay. Fig. 1 1 shows the invariant mass distributions and the corresponding Armenteros-Podolanski plot of the $\Lambda$ and $\bar{\Lambda}$ decay, where the transverse momentum $p_{T}$ is plotted versus the asymmetry of longitudinal momenta of two decay particles.

The $\Lambda$ polarization $P^{\Lambda}$ is measured with respect to its reference axis $L$ (longitudinal) or $T$ (transverse), where $L$ is the momentum vector of the virtual photon and $T$ is the final transverse quark polarization vector in the normal to the $\mu-\mu^{\prime}$ scattering plane. $P^{\Lambda}$ is accessible through the angular distribution of the parity violating weak decay in the $\Lambda$ rest frame by

$$
\frac{d N}{d \cos \theta}=\frac{N}{2}\left(1 \pm \alpha P^{\Lambda} \cos \theta\right)
$$

where $N$ is the number of produced $\Lambda$ hyperons, $\theta$ is the decay angle of the proton with respect to the reference axis. $\alpha= \pm 0.642 \pm 0.013$ is the analyzing power of the parity violating $\Lambda(\bar{\Lambda})$ decay. Due to acceptance effects of the apparatus $P^{\Lambda}$ can not be directly extracted from the experimental data. Two different approaches have been introduced to evaluate these effects. In the measurement of longitudinal $\Lambda$ polarization, the distortion of angular distribution is corrected by using an unpolarized LEPTO MC simulation for $\Lambda$ and $\bar{\Lambda}$ decays. To determine the number of $\Lambda$ hyperon in each $\cos \theta$ bin, a sidebin subtraction method is used. The longitudinal $\Lambda$ polarization $P_{L}^{\Lambda}$ is then obtained from a linear fit of the acceptance corrected angular distribution. In the measurement of transverse $\Lambda$ polarization $P_{T}^{\Lambda}$, the data of the target cells with different polarization and the data taking periods in which the polarization in the cells have been reversed are used in order to cancel acceptance 
effects and leave only the counting rate asymmetry $\varepsilon_{T}(\theta)=\alpha P_{T}^{\Lambda} \cos \theta$ [阿. Finally, the transverse $\Lambda$ polarization is extracted from the slope of $\varepsilon_{T}(\theta)$ distribution.

\section{Results on longitudinal and transverse $\Lambda$ and $\bar{\Lambda}$ polarization}

Averaged on all kinematic variables, the spin transfer to $\Lambda$ is compatible with zero, and no dependence was observed within the present statistical accuracy. In contrast, the spin transfer to $\bar{\Lambda}$ increases with $x_{F}$ reaching values of 0.4. Fig. 目 shows the dependence of spin transfer to $\bar{\Lambda}$ with different model predictions on $x_{F}$ [6]. The sensitivity to the $\bar{s}$ distributions is shown with the comparison of the model predictions obtained with the CTEQ5L and GRV98LO parton distribution functions and based on the SU(6) and Burkardt and Jaffe (BJ) model. The main difference between two parametrization is the allowance of intrinsic $\bar{s}$ contributions in the nucleon. If the $\bar{s}$ quark does not contribute to $\bar{\Lambda}$ polarization, the spin transfer to $\bar{\Lambda}$ vanishes. The CTEQ5L parameterization, in which the intrinsic $\bar{s}$ is 2 times lager than

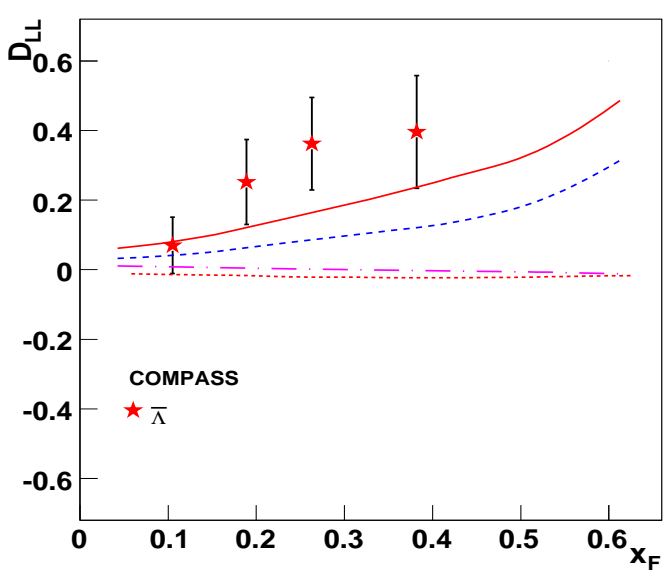

Figure 2: The $x_{F}$ dependence of the longitudinal spin transfer to $\bar{\Lambda}$ : BJ model-CTEQ5L (dotted), SU(6)-GRV98LO (dash-dotted) without $\bar{s}$ contributions, SU(6)-GRV98LO (dashed) and SU(6)-CTEQ5L (solid) with $\bar{s}$ contributions. GRV98LO, describes better the experimental data.

This result should provide an additional information to evaluate the $\bar{s}$ distributions in the nucleon. In Fig. 3 , the $x_{B j}$ dependence of $\Lambda$ and $\bar{\Lambda}$ polarization on the target polarization is shown. Here $P_{-}^{\Lambda}$ is the $\Lambda$ polarization when the target polarization is opposite to the beam polarization and $P_{+}^{\Lambda}$ is the polarization when the target and beam polarization are same. No significant dependence on the target polarization was observed, neither for $\Lambda$ nor for $\bar{\Lambda}$. The exerimental precision has to be increased and the theoretical uncertainties have to be clarified to improve the interpretation of the measurement.

Global fits on the Collins asymmetries for protons (HERMES) and deuteron (COMPASS) have allowed a first extraction of the transversity distributions, showing that $\Delta_{T} u$ and $\Delta_{T} d$ are almost equal in size and shape but opposite in sign [7]. The transverse $\Lambda$ and $\bar{\Lambda}$ polarizations are shown as a function of $x_{B j}$ and $z$ in Fig. 4. Both $\Lambda$ and $\bar{\Lambda}$ show no significant deviation from zero in whole explored range $0 \leq x_{B j} \leq 0.1$. The measured zero value for $P_{T}^{\Lambda}$ might come from the smallness of transversity in the available $x_{B j}$ region, or by the fact that the polarized fragmentation functions are too small in the COMPASS range. If $\Delta_{T} D_{q}^{\Lambda}(z)$ are close to zero, even a larger $\Delta_{T} q\left(x_{B j}\right)$ would be difficult to measure since the spin correlation between a polarized quark and polarized $\Lambda$ hyperon is proportional to the product of $\Delta_{T} q\left(x_{B j}\right)$ and $\Delta_{T} D_{q}^{\Lambda}(z)$. In order to get further informations on the transversity from the measurement of transverse $\Lambda$ polarization, the limited statistics needs to be improved in the valence region, where the transversity distributions are expected to be sizeable. The continued data taking in 2010 with a transversely polarized proton target will provide deeper insight in $\Delta_{T} q\left(x_{B j}\right)$ and $\Delta_{T} D_{q}^{\Lambda}(z)$. 

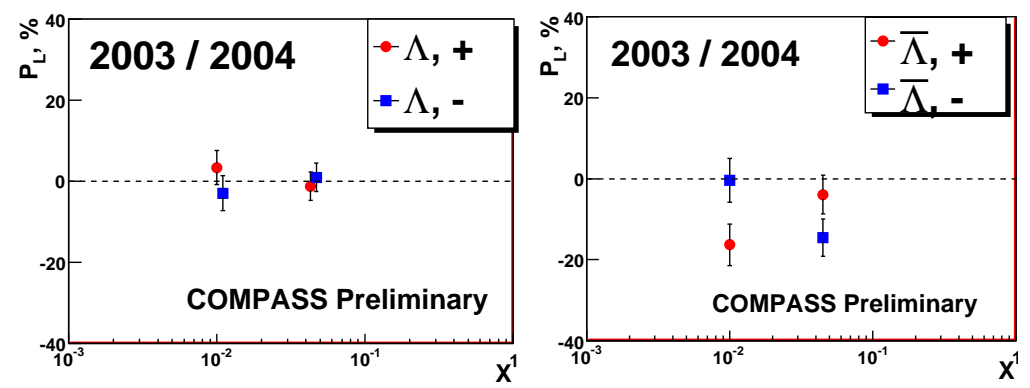

Figure 3: The $x_{B j}$ dependence of longitudinal $\Lambda$ and $\bar{\Lambda}$ polarization for different target polarization.
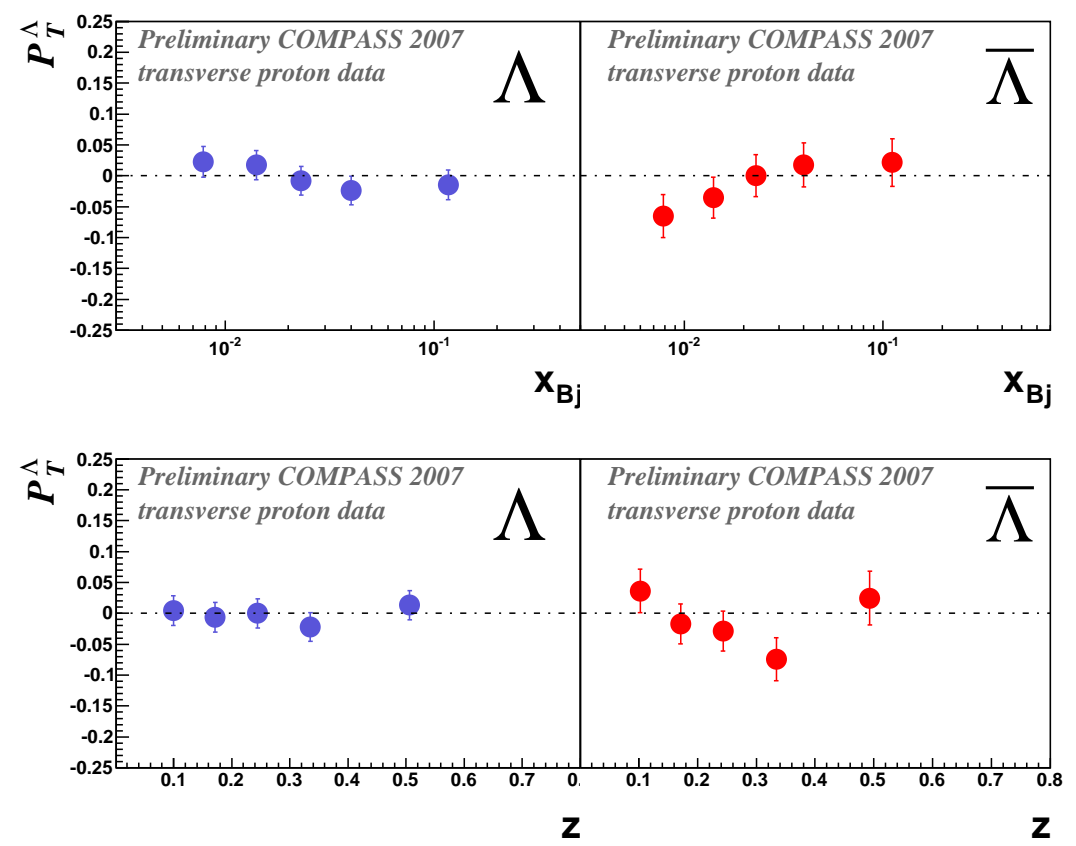

Figure 4: Transverse $\Lambda$ and $\bar{\Lambda}$ polarizations with a transversly polarized proton target in $Q^{2}>1(\mathrm{GeV} / c)^{2}$.

\section{References}

[1] P. Abbon et al., COMPASS Experiment at CERN, Nucl. Inst. Meth. A 577, 455 (2007).

[2] J. Ellis, D. Kharzeev, A. Kotzinian, The proton spin puzzle and $\Lambda$ polarization in deep-inelastic scattering, Z. Physik C 69, 467 (1996).

[3] M. Anselmino, Transversity and Lambda polarization, Proceeding of the Workshop on Future Physics at COMPASS, Sept. 26-27, 2002, CERN.

[4] M. Anselmino et al., $\Lambda$ and $\bar{\Lambda}$ polarization in polarized DIS, Phys. Lett. B 481, 253 (2000).

[5] A. Ferrero, Lambda asymmetries, Proceeding of Transversity 2005, Sept. 7-10, 2005, Como, Italy.

[6] M. Alekseev et al., Measurement of the Longitudinal Spin Transfer to $\Lambda$ and $\bar{\Lambda}$ Hyperons in Polarised Muon DIS, Eur. Phys. J. C 64, 171 (2009)

[7] M. Anselmino et al., Transversity and Collins functions from SIDIS and $e^{+} e^{-}$data, Phys. Rev. D 75 054032 (2007) [hep-ph/ $0701006 \mathrm{v} 3]$. 